\title{
Bilingual Children's Language Development: The Influence of Parental Strategic Input on Vocabulary Development
}

\author{
Yaoruo $\mathrm{Xu}^{1,{ }^{,+}}$Nanyu Zhang ${ }^{2,+}$, Zimeng Zhan ${ }^{3}$, Tianyue Zhang ${ }^{4}$, Chenyu $\mathrm{Fu}^{5}$ \\ ${ }^{1}$ Wenzheng College of Soochow University, Suzhou, 215000,China, yx57@nau.edu \\ ${ }^{2}$ La Salle College Preparatory High School, Pasadena, 91107 USA, mary334515@gmail.com \\ ${ }^{3}$ Lauriston Girls'School, Melbourne, 3000 Australia, m18033888066@163.com \\ ${ }^{4}$ YXUETONG Education, Shanghai, 200000 China, ttzhang123@gmail.com \\ ${ }^{5}$ Hangzhou NO.4 international high school, Hangzhou, 310000 China,fuffu0322@163.com \\ *Corresponding author. Email: yx57@nau.edu \\ + They are both first authors.
}

\begin{abstract}
Bilingual development of children who grow up in dual language environment is often the biggest concern of their parents. In bilingual families, most children grow up to speak the majority language very well, but some may have problems using the minority language, which is usually their ethnic language. However, there are also some children who managed to master both languages well when they grow up, which leads to the following questions: why do some bilingual children can successfully master two languages, while others can't? What are their differences? And what are their methods? Last but not least, what methods can help children who grow up in a bilingual environment to better develop their bilingual learning? This is the topic to be discussed in this paper. We would record the exposure time of children in two languages, and analyze the data to explore the impact of language exposure time on children's bilingual learning. We would also use Peabody Picture Vocabulary Test to compare the receptive vocabulary size of children in families who use two different types of language learning methods, one is the "one-language-one-parent" method, and the other is the "two-parent-one-language" approach, so as to explore the effectiveness of these two different learning methods for bilingual learning and development. We expected the "two-parents-one-language" method to be more effective. The significance of this study is to help bilingual children to find better ways to learn their two languages. It also provides a reference for parents and educators who are anxious to find suitable learning methods. This study can help them objectively deal with the experimental results and choose more appropriate methods for their children.
\end{abstract}

Keywords: bilingual language development, parental strategic, "one-parent-one-language”, "two-parentsone-language”, majority language, minority language, exposure time’, Peabody Picture Vocabulary Test.

\section{INTRODUCTION}

Children who grow up in bilingual environments are not necessarily fluent in both languages. Their development of these two languages may be unbalanced, with a better command of language $X$ and a relatively weaker language Y. Usually, they speak the majority language well rather than their minority language. The majority language refers to the language that mostly used in the place they live (country, community, etc.), while the minority language usually refers to their family language. As Fillmore (2000) argued, if children who are born in a bilingual environment don't speak their family language well, which is usually the ethnic language of parents that they are most comfortable with, it may have a negative impact on the intimacy between parents and children. Both parents and children may be upset when they have an inadequate fluency in their family language for intimate and deeper communication. In addition, bilingual skill is now increasingly needed in numerous working fields. If bilingual children could develop high levels of verbal and related literacy skills in both languages, they are more likely to succeed in their future career. Thus, the value of developing adequate bilingual skills for children has raised more concerns to families and educators, especially when taking the continuing 
immigration and rising births into immigrant families into consideration. There is an urgent need to deal with this imbalanced language development and help bilingual children succeed in learning both languages.

\section{BACKGROUND}

Several previous studies have focused on the patterns of parental language input and children's bilingual development. Although many bilingual children have been exposed to both languages at home since infancy, the effects of the majority language are observed before they enter school [1]. In most cases, bilingual children show a better language skill in the majority language when comparing the majority and minority languages are compared. The use of parental language has been identified as a considerable contributing factor in research on intergenerational language maintenance and loss [2]. Juan-Garau and Perez-Vidal found in their study that the extent to which parents use the minority language at home has a significant effect on their children's ability to communicate in two languages [3].

Specialists and educators proposed the "one-parentone-language" approach to bilingual families, a method in which one parent uses the majority language and the other parent uses the minority language to communicate with their children. However, MacLeod et al studied children who live in German and French speaking families and found that the acquisition of two languages in children in this context where parents speak both languages at home is not balanced, "despite early and consistent exposure to German, this language is not keeping pace with the majority language, in this case French" (p.7) [1]. The "one-parent-one-language" approach does not seem to have promoted children's balanced development of French and German.

Similarly, Siren has found that if both languages are used at home, the child's chances of being actively bilingual are slim. However, the chances are greater if only the minority language is used at home. "Being actively bilingual" here means that the child has the ability to actively use both languages. As noted by De Houwer [4,5], bilingual families may need to explore other ways to support children's bilingual language development. While previous research has looked into the influence of parents on bilingual children's language development, and have assessed the implications of the "one-parent-one-language" approach, there are not many studies in existing literature that discuss the approach of both parents speaking only the minority language. The influence of this approach may require more in-depth research.

\section{PROPOSED STUDY}

This study will focus on the "two-parent-onelanguage" approach, which both parents speak only the minority language at home. We will target the following research questions in our study: 1) how will the "twoparent-one-language" affect the time of children being exposed to both majority and minority languages respectively compared to the "one-parent-one-language" approach? 2) what are some influences of this approach on the vocabulary size of children as opposed to those who are raised in a family where only one parent speaks the minority language?

There are two possible alternatives to the first specific question. The first alternative is that the "two-parent-onelanguage" approach would significantly vary the amount of exposure time to the majority language because children live in an environment where only the minority language is spoken, and they are asked to use only this language. The frequency of their listening and using the minority language has therefore increased. The second alternative is that this approach would not significantly vary their amount of exposure time to the majority language, since children might be immersed in the majority language environment for a longer period of time outside home. The rationale behind is that although the time children exposed to the minority language will be increased through this approach, the corresponding decrease in the exposure time of the majority language is insignificant from an overall perspective.

For the second specific question, a plausible alternative is that the "two-parent-one-language" approach would significantly vary children's vocabulary size regarding the two languages, because parents from this background would grant access to a rather limited word bank in the majority language but a larger vocabulary of the minority language. Therefore, children tend to have larger vocabulary size in the minority language rather than the majority language in this case. However, the other possible alternative can be that children's vocabulary size would not vary significantly because they can also access other resources that provide them with many vocabularies in the majority language such as reading materials, news, and games. It is expected that they could balance the vocabulary of the two languages

\section{METHOD}

\subsection{Participant}

In this study, twenty bilingual children aged 9-10 who are born in the United States will be selected as our samples. In this case, English would be their majority language and Chinese would be their minority language. All of the chosen bilingual children would be exposed to both English and Chinese from birth and would all go to regular elementary school to receive same standard education in English. None of them would show any cognitive and/or physical disabilities. The reason we choose children ranging from $9-10$ is because that all children around nine/ten years old would all have gained 
a basic amount of vocabulary knowledge in both languages, either from home or from school, but not to the extent where the vocabulary size is too hard to measure. All the children selected would be the only child of a family, and none of their families would hire a caretaker. This prevents some irrelevant factors that would impact the children's language ability within home and keeps our study focused only on parental input on bilingual children's language use. Of the twenty children, ten will be from families that use the "one-parent-onelanguage" approach (Approach1), and the other ten will be from families that use the "two-parent-one-language" approach (Approach 2). This sample size of participants is decided based on the study of MacLeod et al, which recruited eleven bilingual children for their study [1].

\subsection{Material}

The study materials we will use include audio recording devices for recording the total amount of exposure time for each language, a questionnaire for parents to answer (see details in the Appendix), designed basing on the questionnaire used in MacLeod et al, and the Peabody Picture Vocabulary Test (PPVT) measuring children's receptive vocabulary in both languages $[1,6]$. The recording device will be given to the children to bring around all day except for their sleeping time. To be more specific, we would be using the LENA recording device as used by Zimmerman [7]. This device weighs about 70 grams and can record up to 16 hours, making it easy for children to carry along throughout the day. To explain the PPVT test, it contains 175 stimulus words and 175 corresponding image plates to match the words. Each image plate has 4 drawings, and only one of them represents the best meaning of the corresponding stimulus word. Children are expected to listen to the word uttered by the interviewer and then choose a corresponding image that best represents the meaning of the word they heard.

\subsection{Procedure}

At the start of the experiment, each child will be asked to wear the LENA recording device for three days and only to take it off during sleeping time. When the threeday recording is over, we will collect data from the recording and calculate the total exposure time of children in each language. These audio data will be divided into multiple different five-minute windows, or intervals, and we would determine if each window is primarily English, Chinese, both, or none of the two languages. When all the five-minute windows are categorized, we will calculate the proportions of each section and save the data for future analysis.

In addition, we will use the PPVT test to measure children's vocabulary size in both English and Chinese. Children will be tested in each language respectively.
This test requires children to identify the image that best matches with the word said by the evaluator. The evaluator will be continuously giving children different words until they mismatch a word or answer eight consecutive words correctly. This will then determine if the testing precede forward or backwards. After finishing the set of words, a raw score is calculated. The test results will then be collected and we will figure out the vocabulary level of the child based their scores. After the children take the test, our questionnaire will also be given out. We require parents to take the questionnaire at the end of the whole process in order to prevent parents from purposely adhering to their response on the questionnaire. (If parents respond that they spent 12 hours speaking a language on a typical workday to the child, during the process of the experiment, they may subconsciously control the total amount of children's exposure time in a language depending on their response). Such thing will hold us back from collecting the real and valid data.

\section{RESULTS AND DISCUSSION}

This research answers the question of how much exposure of both the majority language (English) and the minority language (Chinese) are received by children under the different approaches, and how do these different methods influence their vocabulary size. To analyze the amount of language exposure toward the participants, we would calculate the time of each language spoken toward the child by the parents during these three days and ignore the time of other people's speaking. This will be using the five-minute windows we divided and categorized. The percentage of the time each language each child hears from their parent will be calculated as well as the overall percentage of the time each language is directed toward the children. Using this result, we can determine the overall increase compared to Approach 1, if there is any, in the exposure time of the minority language when the family uses Approach 2. This increase of language exposure time will then be matched with each child's PPVT scores to determine if more exposure time is actually helpful in building children's vocabulary. Furthermore, the questionnaires that are given out for each family to answer will help us to validate our results in the measurement of bilingual language exposure time, but other results from the questionnaire will not be used in present study.

In addition to the audio recordings and questionnaires, both English and Chinese vocabulary test scores of each child will be analyzed, specifically comparing the average scores of children under the "oneparent-one-language" method with children who follow the "two-parent-one-language" method. If children using Approach 2 generally receive a better score on the minority language than children using Approach 1 along with a similar performance on the majority language, then this proves that the "two-parent-one-language" method will help bilingual children to learn the minority 
languages better. In this case, the increase in the input of the minority language may help to strengthen this language's development, thus leading to a better balance in bilingual abilities. If such a conclusion is proven to be true, the "two-parent-one-language" method will be a better and more efficient way for bilingual families to adapt in order to support their child's bilingual development. Contrarily, if the vocabulary scores of the minority language of Approach 2 children does not have a significant difference compared to the scores of Approach 1 children, then the "two-parent-onelanguage" approach is not better than the "one-parentone-language" approach.

\section{CONCLUSION}

Overall, this study concentrates on the impact of parental input on bilingual children. Therefore, we excluded many social factors such as the exposure time of different languages at school, the influence of being exposed to readings, $\mathrm{TV}$-watching and games in different languages on children, and the influence of children's social interaction (with babysitters or friends) on the development of these two languages, etc. In addition, our study only focuses on one-child families, and ignored the influence of alternative family structures on children's bilingual development. The awareness of carrying an audio device also may cause some degree of psychological pressures and uneasiness to the children which could affect their daily language use. Another limitation is that we cannot have a perfect measurement of the vocabulary size of each child to explore their language development in both English and Chinese.

In future studies, we would take social factors that mentioned above into considerations. We could also look at the effects of different family structures on children's bilingual development (e.g., families with two or more minors and examine their impacts upon each other). In addition, we can further analyze the data generated in this research such as looking into whether the overall amount of children's exposure time to languages is relevant to their vocabulary size and how much the children themselves use these different languages at home. And besides from seeing if the increase in minority language input from parents would help increase children's vocabulary in minority language, we could also compare the language exposure time and the test scores of children to answer the question of how much more exposure time will be needed to increase a child's vocabulary size to a certain level. This question brings the research to a deeper level which could not be simply answered by this study. A further research needs to be designed in order to provide a valid answer.

\section{Appendix}

Questionnaire

1. In which language do the following speakers address the child: mother, father, other?

2. How many hours does the child spend in the presence of these speakers on a typical weekday and over a typical weekend?

3. How do the child's expressive and receptive language abilities rate on a five-point scale (1=weak; $2=$ below normal; 3=age appropriate; $4=$ above normal; $5=$ advanced $)$ ?

4. Briefly describe the child's social environment.

\section{REFERENCES}

[1] MacLeod, A., Fabiano-Smith, L., Boegner-Pagé, S., \& Fontolliet, S. (2013). Simultaneous bilingual language acquisition: The role of parental input on receptive vocabulary development. Child Language Teaching and Therapy, 29(1), 131-142. https://doi.org/10.1177/0265659012466862

[2] Kenji, H., \& D'andrea, D. (1992). Some Properties of Bilingual Maintenance and Loss in Mexican Background High-School Students. Applied Linguistics, 13(1), 72-99. https://doi.org/10.1093/applin/13.1.72

[3] Juan-Garau, Maria., \& Pérez-Vidal, Carmen. (2001). Mixing and pragmatic parental strategies in early bilingual acquisition. Journal of Child Language, $28(1)$ 59-86. https://doi.org/10.1017/s0305000900004591

[4] De Houwer, Annick. (2007). Parental language input patterns and children's bilingual use. Applied Psycholinguistics, 28(3), 411-424. https://doi.org/10.1017/s0142716407070221

[5] Sirén, U. (1991). Minority Language Transmission in Early Childhood. Parental intention and language use. Unpublished doctoral dissertation, Stockholm University, Institute of International Education.

[6] Peabody Picture Vocabulary Test - Revised (PPVTR) | National Longitudinal Surveys. (n.d.). National Longitudinal Surveys. https://www.nlsinfo.org/content/cohorts/nlsy79children/topical-guide/assessments/peabodypicture-vocabulary-test-revised

[7] Zimmerman, F. J., Gilkerson, J., Richards, J. A., Christakis, D. A., Xu, D., Gray, S., \& Yapanel, U. (2009). Teaching by listening: the importance of adult-child conversations to language development.

Pediatrics, 124(1), 342-349. 